\title{
Perfluorinated compounds in maternal serum and cord blood from selected areas of South Africa: results of a pilot study $\dagger$
}

\author{
Linda Hanssen, ${ }^{* a b}$ Halina Röllin, ${ }^{c d}$ Jon Øyvind Odland, ${ }^{a}$ Morten K. Moe ${ }^{b}$ and Torkjel M. Sandanger ${ }^{a b}$ \\ Received 21st December 2009, Accepted 9th March 2010 \\ First published as an Advance Article on the web 28th April 2010 \\ DOI: $10.1039 / b 924420 d$
}

\begin{abstract}
There is limited information about both environmental and human perfluorinated compounds (PFCs) concentrations in the southern hemisphere, and for the first time, concentrations of these compounds are reported in maternal serum and cord blood of South African women. The majority of the participants were of African Black ethnicity, with a similar socioeconomic status. In maternal serum perfluorooctane sulfonate (PFOS) was found to be the most abundant PFC (1.6 $\left.\mathrm{ng} \mathrm{mL}^{-1}\right)$, followed by perfluorooctanoate (PFOA: $1.3 \mathrm{ng} \mathrm{mL}^{-1}$ ) and perfluorohexane sulfonate (PFHxS: $0.5 \mathrm{ng} \mathrm{mL}^{-1}$ ); however, in cord blood PFOA was the most abundant compound $\left(1.3 \mathrm{ng} \mathrm{mL}^{-1}\right)$ followed by PFOS $\left(0.7 \mathrm{ng} \mathrm{mL}^{-1}\right)$ and PFHxS $\left(0.3 \mathrm{ng} \mathrm{mL}^{-1}\right)$. Linear PFOS constituted $58 \%$ of the sum of PFOS, comparable with a reported percentage from Australia. Differences in PFC concentrations between communities were found, with the highest concentrations in urban and semi-urban areas. The median maternal PFOS concentration was lower than has been reported in other studies, whereas the PFOA concentration was the same. This clearly indicates that the exposure pathway is different from the western world. Significant differences in housing quality were observed and the urban and sub-urban community had the highest living and housing standards. Possible exposure pathways could be different from those elucidated in the western world with the exception of the urban community in our study that showed higher living standards in general and easier access to modern consumer products.
\end{abstract}

\section{Introduction}

Perfluorinated or polyfluoroalkyl compounds (PFCs) are synthetic fluorinated organic compounds used in a number of consumer products and industrial applications. The chemical structure of the PFCs, a hydrophobic alkyl chain and a hydrophilic functional group, gives these compounds unique properties, which have been used in various applications such as fire-fighting foams, mining and oil well surfactants, in the leather and textile industry, as consumer products for cleaning and

a Department of Community Medicine, University of Tromsø, 9037 Tromsø, Norway.E-mail: linda.hanssen@uit.no; Tel: +4792887291

${ }^{b}$ Norwegian Institute for Air Research, the Polar Environmental Centre, 9296 Tromso, Norway

'Medical Research Council, Johannesburg, South Africa

${ }^{d}$ School of Health Systems and Public Health, University of Pretoria, Pretoria, South Africa

$\uparrow$ Electronic supplementary information (ESI) available: Table S1: socioeconomic and housing characteristics of participants by site; Fig. S1: chromatogram of unspecified branched and linear isomers of PFOS. See DOI: 10.1039/b924420d polishing, and as an insecticide. ${ }^{1,2}$ Emissions of PFCs into the environment, in the northern hemisphere, come mostly from fluoropolymer manufacturing; air and water are the main transport routes to remote places. ${ }^{3}$ Recent monitoring studies have shown the presence of PFCs world wide, in various environmental compartments, including wildlife and humans, ${ }^{4-6}$ and perfluorooctane sulfonate (PFOS) is classified as a persistent organic pollutant (POP) according to the Stockholm convention of $2009 .^{7}$

PFOS and perfluorooctanoate (PFOA) are two of the most widely detected and studied compounds in the PFC class, and the main PFCs found in animal tissue and human plasma. ${ }^{8}$ Among wildlife, the highest concentrations of PFOS have been found in mink and polar bears. ${ }^{4,9}$ In humans, the highest concentrations have been found among occupationally exposed workers. ${ }^{10}$ The tissue distribution of PFCs in human and other species is different from that observed for other POPs. ${ }^{11}$ The highest PFC concentrations are found in protein-rich organs, such as the liver and spleen, ${ }^{8}$ and blood as PFCs are bound to albumin. ${ }^{12}$ The half-lives of PFCs are species and compound dependent, and for

\section{Environmental impact}

There have been several reports on perfluorinated compounds (PFCs) concentrations in human plasma around the world, however there has been limited information from the southern hemisphere and no reports from Africa. To our knowledge this is the first study that reports PFC concentrations in plasma from delivering women and cord blood samples from South Africa. This work contributes to improved knowledge about global PFC distribution. The distribution of PFCs between six sites covering rural and urban residences, was investigated and the highest concentrations were found in urban/semi urban areas. Paired maternal-cord samples gave additional information about maternal transfer of PFCs and PFOS isomers to the foetus 
some species there are also observed gender differences in elimination of PFOA. ${ }^{8}$ In humans, the half-lives (geometric means) of PFOS, PFOA and perfluorohexane sulfonate (PFHxS) have been reported to be 5.4, 3.5 and 7.3 years, respectively. ${ }^{13}$

Both PFOS and PFOA are associated with hepatotoxicity and liver enlargement in rodents and non-human primates. ${ }^{14,15}$ They are considered to have the potential to cause developmental toxicity in animals. ${ }^{16}$ In utero exposure to PFOA in mice had effects at low doses, such as lower body weight and increased insulin and leptin concentrations when measured in animals during mid-life. ${ }^{17}$ Lau et al. have presented an overview of the developmental toxicity and recent advances in the toxicology and mode of action for perfluoroalkyl acids. ${ }^{8}$

Inoue et $a l^{18}$ were the first to report the presence of PFCs in human maternal and cord blood samples, and several other studies followed. ${ }^{19-21}$ Furthermore, recent epidemiological studies in Denmark, USA and Japan ${ }^{21-23}$ have investigated possible adverse effects of PFCs on the foetus. In the Danish cohort they found an inverse association between maternal PFOA concentration and birth weight. A small inverse association between both PFOS and PFOA and birth weight and size was reported in the American study. In the Japanese study, there was no correlation between maternal PFOA plasma concentration and birthweight, but they found that in utero exposure to relatively low levels of PFOS correlated negatively with birthweight. These studies have recently been reviewed by Olsen et al. ${ }^{24}$ Even Olsen concluded that though there were inconsistent associations reported for several different birth outcomes the authors of the published studies had been appropriately cautious in the interpretation of their analyses. In future studies they suggest that plasma volume expansion during pregnancy should be accounted for.

According to Prevedouros et al. no significant use and production of PFCs are known to have occurred in the southern hemisphere. $^{3,25}$ It is thought that the atmospheric and aquatic mass exchange for PFCs between the two hemispheres is limited, ${ }^{26}$ and environmental data from air and water measurement indicate no significant long-range atmospheric transport of neutral volatile PFCs emitted in the northern hemisphere. ${ }^{27,28}$ In addition there is no comprehensive information about PFC use in South Africa, and local sources could be imported consumer products, insecticides formulations against termites and mining surfactants. There is limited information about human PFC concentrations in the southern hemisphere. Two human studies from Australia ${ }^{25,29}$ reported PFC concentrations that were comparable to the northern hemisphere, and in one study from Brazil, the concentrations were 5-10 times lower than levels found in the sera of donors in the United States. ${ }^{30}$

The aim of this study was to assess PFC concentrations in maternal serum and cord blood in delivering women in selected areas of South Africa in relation to age and parity. Additional information about paired maternal-cord concentrations and PFOS isomers is also presented.

\section{Materials and methods}

\section{Community differences}

Seven communities were previously selected, ${ }^{31}$ of these the following six were included in the present PFC study. Selected communities differed in the type of environmental pollution and all had a provincial delivery hospital serving particular community. Fig. 1 shows the geographical location of each community within South Africa. The urban community (Site 2) is the large city of Johannesburg with extensive industrial activities. The industrial community (Site 3 ) is dominated by coal mining and stainless steel production. The Atlantic Ocean community (Site 4) is situated in the Western Cape and is known for its fishing and fish processing industry. The mining site (Site 5) is a small town where extensive gold mining takes place. The coastal village (Site 6) on the Indian Ocean is malaria endemic, where only subsistence fishing is allowed. The inland site (Site 7) is a small town with little industrial activity about $70 \mathrm{~km}$ away from the Indian Ocean coastal site, but is also malaria endemic. The concentration of toxic metals and persistent organic pollutants in the study population has been reported elsewhere. ${ }^{31,32}$

\section{Recruitment of participants and informed consent}

Ethics clearance certificate (Protocol Number M040314) was granted for the study by the Committee for Research on Human Subjects of the University of the Witwatersrand, Johannesburg, South Africa. In addition, informed written consent was obtained from each participant prior to inclusion in the study.

Participants were recruited among women who presented for delivery at the hospital by the health worker on duty and a trained researcher who briefly explained the objective of the study and distributed a detailed information sheet about the project, written in simple language. Women who volunteered to participate signed an informed consent form and agreed to donate blood before delivery and cord blood post-partum, as well as to answer a socioeconomic questionnaire and to grant research team access to hospital records post-partum. Samples were collected from November 2005 to December 2006.

\section{Sampling procedures}

For each mother, $30 \mathrm{ml}$ of blood was drawn by venous puncture into Vacutainer tubes before delivery, and umbilical cord blood was collected after delivery by a nursing sister, using a Vacutainer disposable system. The samples were centrifuged at the hospital and serum was transferred to pre-rinsed glass tubes before storage at $-20{ }^{\circ} \mathrm{C}$. Great care was taken to prevent contamination of samples during collection and fractionation, all equipment used had previously been tested to avoid contamination of samples. Details about collection of socioeconomic and delivery data are described in a previous publication. ${ }^{32}$

\section{Chemical analysis}

The analytical method used was a slight modification of the published method of Rylander et al. ${ }^{33}$ The main differences were the amount of serum/blood and methanol used. In short, $0.5 \mathrm{~g}$ serum was transferred to a $50 \mathrm{~mL}$ centrifugation tube (Nalgene, Nalge Nunc International, Rochester, NY, USA) and $5 \mathrm{ng}$ of each internal standard $-\left[{ }^{13} \mathrm{C}_{4}\right] \mathrm{PFOA}$ and $\left[{ }^{13} \mathrm{C}_{4}\right] \mathrm{PFOS}$ in methanol (Wellington Laboratories, Guelph, ON, Canada) - were added to the sample before subsequent addition of $4 \mathrm{~g}$ methanol (Lichrosolv, Merck, Darmstadt, Germany). After extraction the 


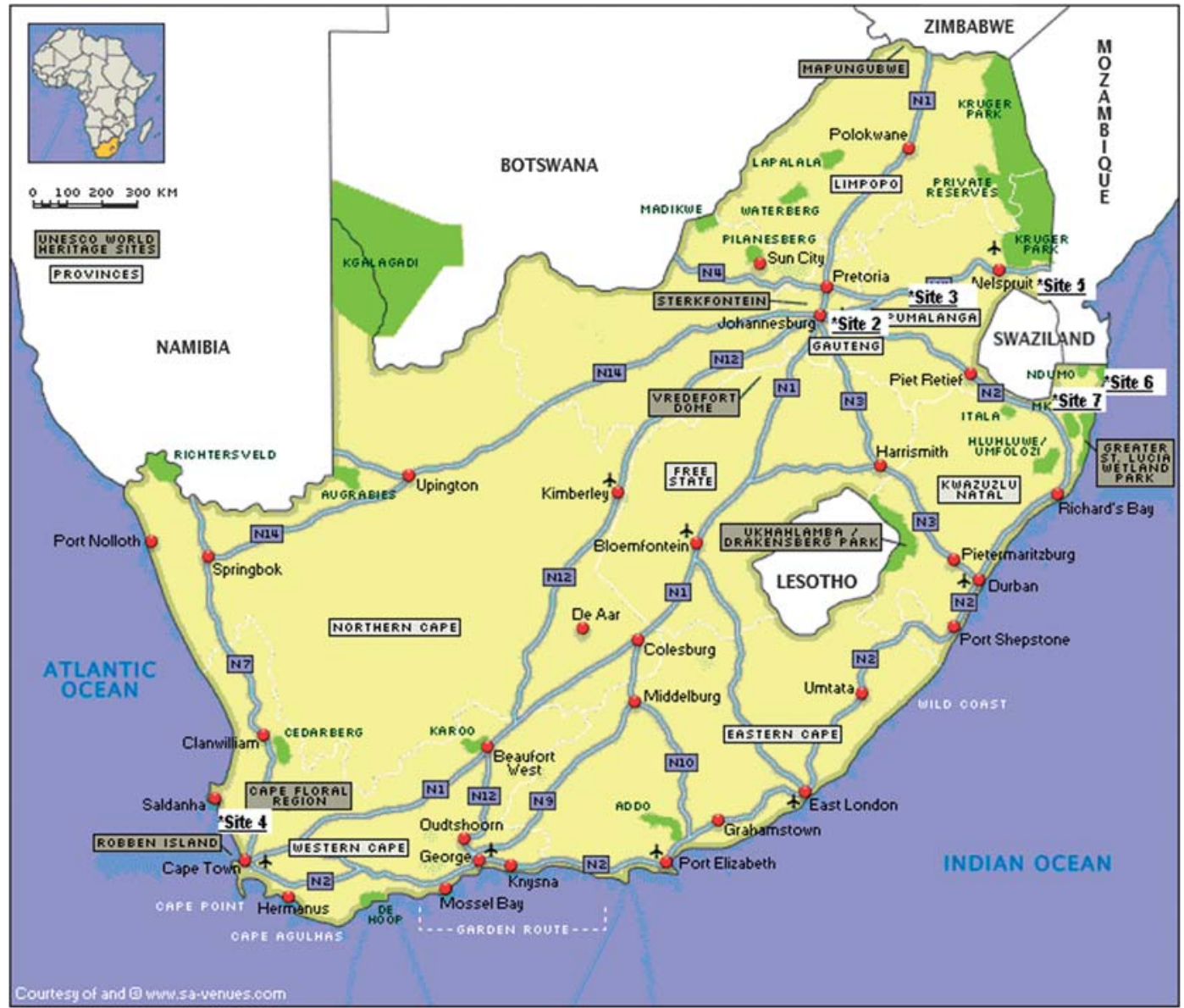

Fig. 1 Geographical positions of study sites within South Africa: Site $2=$ urban (Johannesburg); Site $3=$ industrial; Site $4=$ Atlantic Ocean; Site $5=$ mining; Site $6=$ coastal malaria; Site 7 = inland malaria.

supernatants were transferred to new tubes and the volume reduced to approximately $1 \mathrm{~mL}$ before vortexing the sample with acidified ENVI-Carb 120/400 (Supelco, PN, USA). ${ }^{34}$ Supernatant $(500 \mu \mathrm{L})$ was transferred to a $1.5 \mathrm{ml}$ vial (VWR, West Chester, PN, USA) and mixed with $500 \mu \mathrm{L} 2 \mathrm{mM}$ aqueous ammonium acetate (BDH Laboratory Supplies, UK). Finally, $5 \mathrm{ng}$ 3,5-bis(trifluoromethyl)phenyl acetic acid (BTPA, Wellington Laboratories, Guelph, ON, Canada) was added as recovery standard.

The PFCs were analysed using a QTOFmicro quadropoletime-of-flight (QTOF) mass spectrometer, as described by Rylander et $a l^{33}$ The quantification was conducted using the QuanLynx version 4.0 software from Waters.

\section{Quality control}

The quality of the analysis was checked by regular analysis of blank samples and samples with known PFC concentrations (reference samples) from previous rounds of international calibration programmes. For each batch of 30 samples, two blank samples and two reference samples were prepared. In addition, our laboratory participates regularly in an interlaboratory comparison programme, through the AMAP (Arctic Monitoring Assessment Programme) Ring Test for Persistent Organic Pollutants in Human Serum (three rounds a year), organized by the Institut National de Santé Publique du Québec, Canada. Results from the interlaboratory comparison programme indicate that any uncertainties in our analysis are well within $\pm 30 \%$ of assigned values. The recovery rates of the labelled internal standards varied from $40 \%$ to $120 \%$. The limit of detection (LOD) was automatically calculated by the quantification software. It corresponds to three times that of the signal to noise ratio within each sample, accounting for matrix differences in individual samples. No analytes at concentrations above the LOD were detected in the blank samples. Concentrations of PFCs in all samples were well within the linear range of the instrument.

PFOS was quantified and reported as the sum of the unspecified branched PFOS and linear PFOS. They were chromatographically separated in two peaks and allowed the calculation of area ratios between the two. The branched isomers were not structurally elucidated but rather identified as eluting earlier than the linear isomers as indicated in Figure S1, ESI $\uparrow{ }^{35}$

\section{Statistical analysis}

Statistical analysis was performed using the STATA package, version 10 (StataCorp LP, Texas, USA). Due to non-normal distribution of the concentrations among the participants, the concentrations were log-transformed before the statistical 
Table 1 Perfluorinated compound concentrations $\left(\mathrm{ng} \mathrm{mL}^{-1}\right)$ in the study group, method detection limit (LOD) and percentage linear isomers

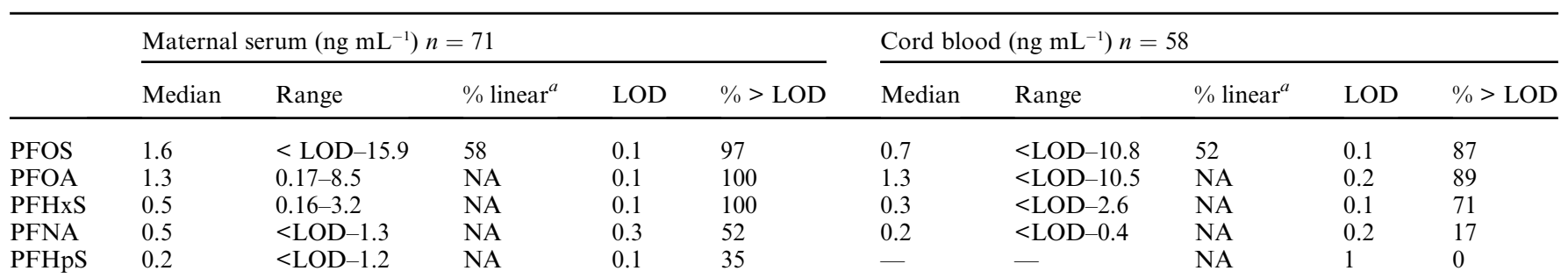

${ }^{a}$ NA, not available, PFOS, perfluorooctane sulfonate; PFOA, perfluorooctanoate; PFHxS, perfluorohexane sulfonate; PFNA, perfluorononanoate; PFHpS, perfluoroheptane sulfonate; \% linear isomers of PFOS, percentage linear isomers of PFOS related to the sum of the linear and branched isomers.

analysis. Only PFOS, PFOA and PFHxS that were detected in more than $70 \%$ of the samples, were further statistically elucidated (Table 1).

The impact of age, parity and place of residence on the measured concentrations of PFOS, PFOA and PFHxS was investigated using linear regression. Model assumptions for the linear models, outliers and influential observations were evaluated using diagnostic plots of the residuals. Parameter estimates $(\beta)$ with $95 \%$ confidence intervals (CIs), $F$ statistics, $R^{2}$ values, Wilcoxon's test estimator $(W)$, Spearman's rank correlation coefficient $(r)$, degrees of freedom (D.F.) and $p$ values are reported in the text. The parameter estimates $(\beta)$ are backtransformed log results which should be interpreted as the number of times the response variable increases/decreases by a 1 unit increase in explanatory variable.

For statistical purposes, in samples where PFC concentrations were equal to or below the LOD, the concentrations were set to $0.5 \times$ LOD. $^{11}$

The cord blood concentrations were corrected for the volume displacement of the blood cells, and were multiplied by a factor of 2 for PFOA, PFHxS and PFOS according to Ehresman et al. ${ }^{36}$ The PFC concentrations were converted from $\mathrm{ng} \mathrm{g}^{-1}$ to $\mathrm{ng} \mathrm{mL}^{-1}$ by multiplying by a factor of 1.03 as a correction of the difference in mass and volume.

\section{Results}

Serum and cord blood were analysed for five PFCs: the median PFOS, PFOA, PFHxS, perfluorononanoate (PFNA) and perfluoroheptane sulfonate (PFHpS) serum concentrations, and their range and percentage linear isomers of PFOS, are presented in Table 1. Maternal PFOS, PFOA and PFHxS serum concentrations, and maternal age and parity are given in Table 2 for each community. A more detailed description of socioeconomic and housing characteristics of participants at each community is presented in the ESI $\uparrow$ (Table S1). ${ }^{31}$ There was no statistical difference in ethnicity of the participants between study communities with the majority being of African Black ethnicity. Most of the women were unemployed, had no previous occupational history and relied mainly on social grants or financial support from their partner and other family members. Self reported household income was highest in the urban, followed by Atlantic and mining communities. The urban community has the highest living and housing standards, comparable to other city dwellers in South Africa.
The correlation coefficient for PFOS and PFOA concentrations in maternal serum was $0.64(p<0.001)$, for PFOS and PFHxS $0.79(p<0.001)$, and for PFOA and PFHxS 0.58 $(p<0.001)$. Correlation coefficients between maternal and cord blood for PFOS, PFOA and PFHxS were $0.88,0.67$ and 0.77 , respectively - all being statistically significant $(p<0.001)$. After volume correction the average maternal : cord ratios for PFOS, PFHxS and PFOA were 2.2 (range 0.5-7.9), 2.1 (range 0.3-9.9) and 1.4 (range $0.2-7.1$ ), respectively.

The results from univariate linear regression analysis for maternal PFOS, PFOA and PFHxS concentration and age were found to be significant only for PFOA $(\beta=1.03, p=0.040, \mathrm{CI}=$ $\left.0.00135-0.05573, R^{2}=0.047\right)$. After adjusting for age and parity, place of residence significantly predicted the PFC concentration: PFOS $\left(F_{7,59}=12.36\right.$, adjusted $\left.R^{2}=0.546, p<0.001\right)$, PFOA $\left(F_{7,59}=5.33\right.$, adjusted $\left.R^{2}=0.315, p<0.001\right)$ and PFHxS $\left(F_{7,59}=\right.$ 12.04 , adjusted $\left.R^{2}=0.539, p<0.001\right)$. PFOS and PFHxS concentrations in maternal serum from mothers living in Indian Ocean (PFOS: $\beta=0.29, p<0.001$; PFHxS: $\beta=0.43, p<0.001$ ), mining (PFOS: $\beta=0.33, p<0.001$; PFHxS: $\beta=0.44, p<0.001$ ) and inland malaria sites (PFOS: $\beta=0.20, p<0.001$; PFHxS: $\beta=$ $0.36 p<0.001$ ) (all predominantly rural) were significantly lower compared with Johannesburg, industrial (PFOS: $\beta=0.81$, $p>0.05$; PFHxS: $\beta=0.98, p>0.05$ ) and Atlantic Ocean sites (PFOS: $\beta=1.33, p>0.05$; PFHxS: $\beta=1.16, p>0.05$ ) (urban and semi-urban sites). Similarly, maternal PFOA concentrations in Indian Ocean $(\beta=0.53, p<0.001)$ and the inland malaria site $(\beta=0.42, p<0.001)$ were significantly lower than in Johannesburg, and industrial $(\beta=1.18, p>0.05)$, Atlantic Ocean $(\beta=$ $1.20, p>0.05)$ and mining sites $(\beta=0.90, p>0.05)$. In Fig. 2 the log-transformed PFOS, PFOA and PFHxS concentrations for each site are presented.

There was a significant difference in the area percentage of linear isomers of PFOS in maternal serum and cord blood, according to Wilcoxon's signed rank $(p<0.05)$.

\section{Discussion}

To the best of our knowledge, this is the first time that PFCs in serum of delivering women and cord blood samples have been measured in South Africa. PFOS was the most abundant compound in maternal serum, followed by PFOA and PFHxS, as opposed to the cord blood, where PFOA dominated, followed by PFOS and PFHxS. This was not observed in earlier studies. The median maternal PFOS concentration was $1.6 \mathrm{ng} \mathrm{mL} \mathrm{mL}^{-1}$, much 


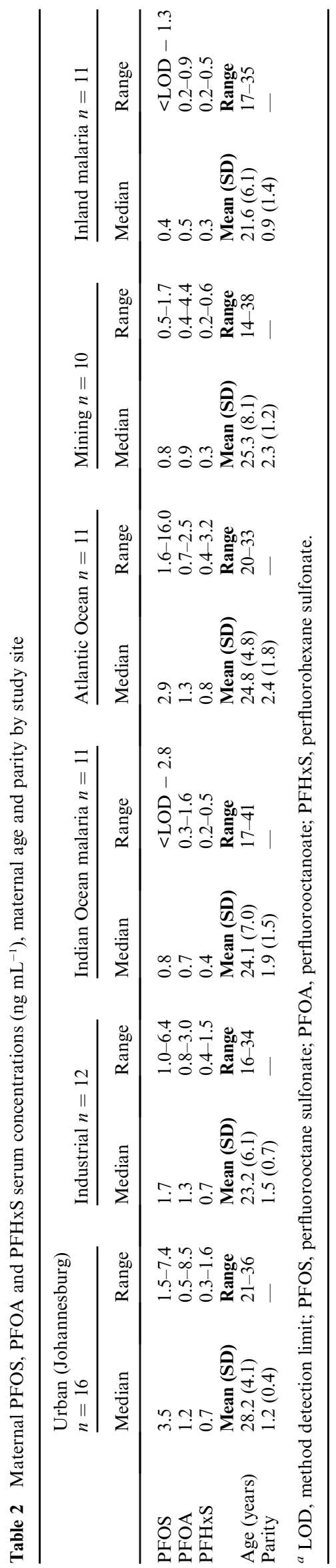

lower than that reported from highly industrialized countries such as Canada, Japan and Germany, where PFOS concentrations were reported to be $14.54 \mathrm{ng} \mathrm{mL}^{-1}, 8.10 \mathrm{ng} \mathrm{mL}^{-1}$ and $13.0 \mathrm{ng} \mathrm{mL}{ }^{-1}$, respectively. ${ }^{18,37,38}$ The median PFOA concentration measured in maternal serum in this study was $1.3 \mathrm{ng} \mathrm{mL}^{-1}$, comparable with concentrations reported in the studies mentioned above where it ranged from not detectable (Japan) to $2.6 \mathrm{ng} \mathrm{mL} \mathrm{m}^{-1}$ (Germany). ${ }^{18,38}$ This is consistent with reported PFOA concentrations in the general population from countries world wide, were PFOA in human sera from Brazil, Colombia and Australia were comparable to those of Europe and North America. ${ }^{39}$ On the other hand, PFOS concentrations vary with the highest concentrations reported in human sera from industrialised countries. ${ }^{30}$ The low PFOS concentration in South Africa could be a result of historically lower exposure and the phase-out of perfluorooctane sulfonylfluoride (PFOS precursor) in 2000-2002. After the phase out of PFOS in 2000-2002, reports have shown a simultaneous decline in PFOS and PFOA concentrations, ${ }^{40}$ indicating that exposure pathways for these compounds were historically linked, ${ }^{39,40}$ even if production of PFOA and various perfluorinated acids continues today. ${ }^{41} \mathrm{~A}$ decline in PFOA concentration has not been observed in Australia. ${ }^{29}$ Due to the paucity of information on PFC content in the environment and other media in the southern hemisphere, it is difficult to assess which factors contribute to the body burden of PFCs in South Africa. Generally, it is understood that food is considered to be the main exposure pathway for PFCs, with air and dust also being considerable contributors. ${ }^{42-44}$

In South Africa, the maternal median PFHxS concentration was found to be $0.5 \mathrm{ng} \mathrm{mL}{ }^{-1}$. Only two studies have reported concentrations for this compound in maternal serum: in Canada $^{37}$, the median concentration was $1.62 \mathrm{ng} \mathrm{mL}^{-1}$ and in Vietnam ${ }^{33} 0.7 \mathrm{ng} / \mathrm{mL}$. In the study from Canada, the researchers also measured PFHxS in cord samples, but found PFHxS in only $20 \%$ of the samples, with a median concentration of $2.07 \mathrm{ng} \mathrm{mL}^{-1}$. In the current study the PFHxS was detected in $71 \%$ of the samples, although the cord concentration was lower, at $0.3 \mathrm{ng} \mathrm{mL}^{-1}$.

The lower maternal : cord ratios for PFOA compared to PFOS and PFHxS indicated that PFOA passes through the placenta more easily than PFOS and PFHxS. This has also been reported by Midasch et al. ${ }^{38}$ where PFOA concentrations in cord blood plasma were higher compared with the maternal plasma. They concluded that PFOA passes the placental membrane unhindered.

The three dominant PFCs in maternal samples correlated, with the strongest correlation being between PFOS and PFHxS ( $r=$ $0.79)$, this is in agreement with other reports. ${ }^{6,42}$ The correlation between PFOS and PFOA and between PFOS and PFHxS concentrations were medium strong $(r=0.64$ and 0.58 , respectively). Several studies from North America and Europe have reported significant correlations between PFOS and PFOA. ${ }^{39}$ Since PFOS cannot convert directly to PFOA and vice versa, it has been suggested that a strong correlation between PFOS and PFOA indicates a co-exposure of these compounds present in various products. ${ }^{30}$ The correlation between PFOA and PFHxS was stronger than that reported for American blood donors ${ }^{6}(r=$ 0.32 ) but weaker than that reported for delivering women from Vietnam $^{33}(r=0.94)$. The differences in correlation may indicate different exposures to this compound. 

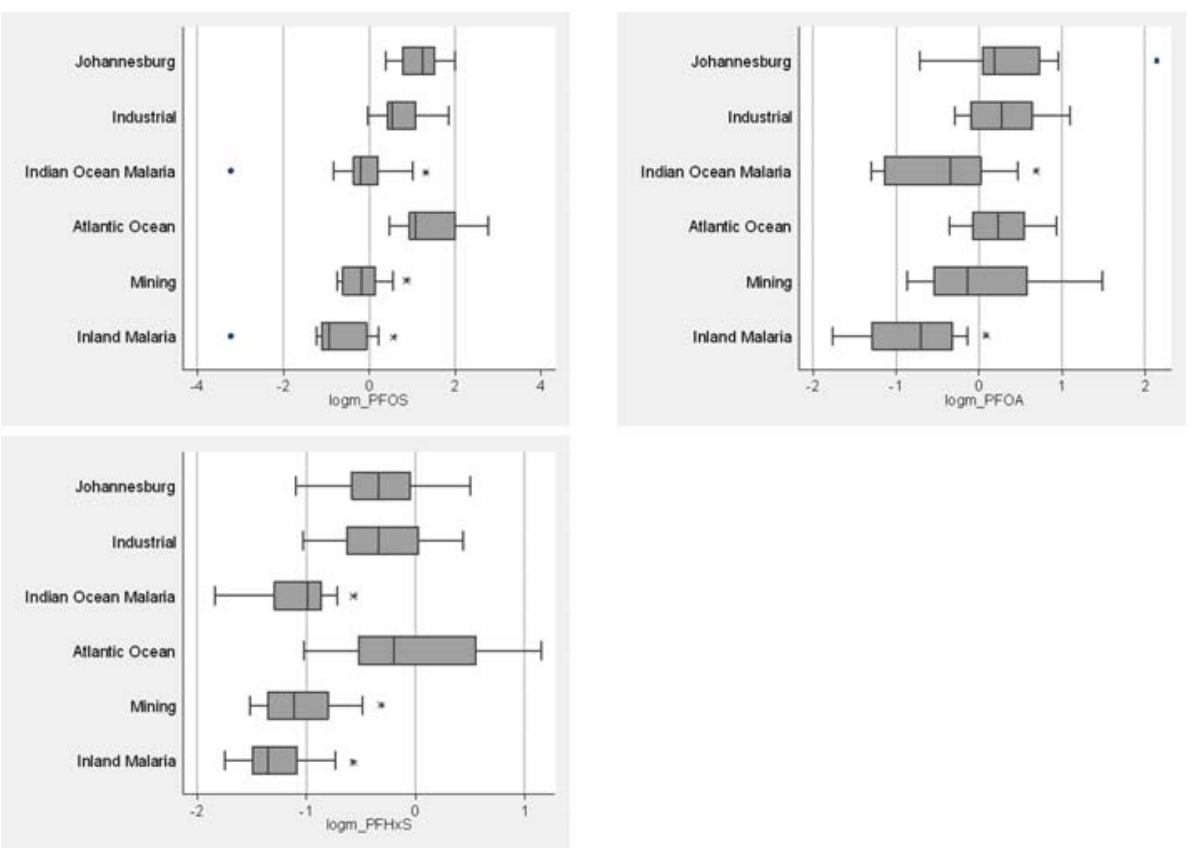

Fig. 2 The distribution of log-transformed maternal concentration of perfluorooctane sulfonate (PFOS), perfluorooctanoate (PFOA) and perfluorohexane sulfonate (PFHxS) from the areas in South Africa selected.

Place of residence can be used to significantly predict maternal PFC concentrations (Fig. 2), similar to the conclusions of von Ehrenstein et al. ${ }^{45}$ Among the places of residence, we found distinct differences in living conditions, diet and housing type. Possible exposure pathways could be different from those elucidated in the western world with the exception of the urban community in our study that showed higher living standards in general and easier access to modern consumer products such as stain resistant furniture, carpets, clothing, fast foods and cosmetics, to name but a few.

The observed percentage of linear isomers of PFOS in maternal serum and cord blood, 58\% and 54\% respectively, is lower than what has been reported for the technical product (70$80 \%){ }^{46}$ In human plasma or serum samples, the reported linear percentage ranged from $58 \%{ }^{35}$ to $83 \%{ }^{33}$ It has been suggested that the increased percentage of branched isomers of PFOS could be explained by longer half-lives compared with linear isomers of PFOS. ${ }^{44}$ This does not, however, agree with the half-lives for different PFOS isomers in rats, ${ }^{41}$ where branched isomers of PFOS, apart from 1-perfluoromethyl PFOS, had shorter halflives than linear isomers. As a result of chromatographic limitations, it was not possible to elucidate the PFOS isomer profile in this study. In a recent study by Ridell et al. ${ }^{47}$ it was found that in two human serum standard reference materials there were dramatically different branched PFOS isomer profiles, with isoPFOS being the most dominant isomer.

The observed difference in ratio could be explained firstly by the branched isomers passing through the placenta more easily than the linear ones and/or, secondly, the branched isomers interacting more strongly with the blood cell fraction than the linear isomers, the observed difference thus being related to matrix.

\section{Conclusions}

This study found that delivering women in South Africa and their babies have been exposed to PFCs, even if there are no known large-scale sources of these compounds in South Africa. The PFOS concentration was lower than has been reported in other studies, but the PFOA concentration was comparable. Differences between communities were observed, with the highest concentrations of PFCs measured in urban areas. The dominant compound in maternal plasma was PFOS. In cord blood, PFOA was dominant due to the differences in transport through the placental membrane. There also seems to be a difference in transport across the placental membrane between branched and linear isomers of PFOS, with branched isomers passing more easily.

Future studies in South Africa could help understand the human expouse to PFCs since there are distinct differences in living conditions between communities.

\section{Acknowledgements}

The authors thank the Arctic Monitoring and Assessment Programme (AMAP), Oslo, Norway; the Nordic Council of Ministers, Copenhagen, Denmark; Northern Norway Regional Health Authority, Bodø, Norway; Norwegian Ministry of Foreign Affairs, Oslo, Norway; and the SA Medical Research Council, Johannesburg, South Africa for financial support of this study. Support from the relevant health departments, maternity sections and staff of hospitals is also acknowledged. Above all, we thank all the mothers who agreed to participate. Special appreciation goes to Anna Helena Falck (NILU) for her analytical work; and research technologist, Mirriam Mogotsi 
(MRC), for her expert research assistance in data and sample collection. We thank the staff of the Analytical Services laboratory of the NIOH, Johannesburg, for processing of the biological samples.

\section{References}

1 R. Renner, Environ. Sci. Technol., 2001, 35, 154A-160A.

2 S. Posner, D. Herzke, P. B. Poulsen and A. Jensen, -, 2007, TA-2354, pp. 1-76.

3 K. Prevedouros, I. T. Cousins, R. C. Buck and S. H. Korzeniowski, Environ. Sci. Technol., 2006, 40, 32-44.

4 J. P. Giesy and K. Kannan, Environ. Sci. Technol., 2001, 35, 13391342.

5 M. Houde, J. W. Martin, R. J. Letcher, K. R. Solomon and D. C. G. Muir, Environ. Sci. Technol., 2006, 40, 3463-3473.

6 G. W. Olsen, T. R. Church, J. P. Miller, J. M. Burris, K. J. Hansen, J. K. Lundberg, J. B. Armitage, R. M. Herron, Z. Medhdizadehkashi, J. B. Nobiletti, E. M. O’Neill, J. H. Mandel and L. R. Zobel, Environ. Health Perspect., 2003, 111, 1892-1901.

7 T. Wang, Y. W. Wang, C. Y. Liao, Y. Q. Cai and G. B. Jiang, Environ. Sci. Technol., 2009, 43, 5171-5175.

8 C. Lau, K. Anitole, C. Hodes, D. Lai, A. Pfahles-Hutchens and J. Seed, Toxicol. Sci., 2007, 99, 366-394.

9 J. W. Martin, K. Kannan, U. Berger, P. D. Voogt, J. Field, J. Franklin, J. P. Giesy, T. Harner, D. C. G. Muir, B. Scott, M. Kaiser, U. JÃărnberg, K. C. Jones, S. A. Mabury, H. Schroeder, M. Simcik, C. Sottani, B. V. Bavel, A. KÃarrman, G. LindstrÃ $\lceil\mathrm{m}$ and S. V. Leeuwen, Environ. Sci. Technol., 2004, 38, 248A-255A.

10 OECD, Co-operationon existing chemicals. Hazard assessment of perfluorooctanesulfonate (PFOS) and its salts, OECD, Paris, 2002.

11 A. Karrman, B. van Bavel, U. Jarnberg, L. Hardell and G. Lindstrom, Chemosphere, 2006, 64, 1582-1591.

12 D. J. Luebker, K. J. Hansen, N. M. Bass, J. L. Butenhoff and A. M. Seacat, Toxicology, 2002, 176, 175-185.

13 G. W. Olsen, J. M. Burris, D. J. Ehresman, J. W. Froehlich, A. M. Seacat, J. L. Butenhoff and L. R. Zobel, Environ. Health Perspect., 2007, 115, 1298-1305.

14 A. M. Seacat, P. J. Thomford, K. J. Hansen, G. W. Olsen, M. T. Case and J. L. Butenhoff, Toxicol. Sci., 2002, 68, 249-264.

15 A. M. Seacat, P. J. Thomford, K. J. Hansen, L. A. Clemen, S. R. Eldridge, C. R. Elcombe and J. L. Butenhoff, Toxicology, 2003, 192, 263-264.

16 G. L. Kennedy, J. L. Butenhoff, G. W. Olsen, J. C. O'Connor, A. M. Seacat, R. G. Perkins, L. B. Biegel, S. R. Murphy and D. G. Farrar, Crit. Rev. Toxicol., 2004, 34, 351-384.

17 E. P. Hines, S. S. White, J. P. Stanko, E. A. Gibbs-Flournoy, C. Lau and S. E. Fenton, Mol. Cell. Endocrinol., 2009, 304, 97-105.

18 K. Inoue, F. Okada, R. Ito, S. Kato, S. Sasaki, S. Nakajima, A. Uno, Y. Saijo, F. Sata, Y. Yoshimura, R. Kishi and H. Nakazawa, Environ. Health Perspect., 2004, 112, 1204-1207.

19 B. Apelberg, A. Calafat, J. Herbstman, F. Witter, R. Halden, Z. Kuklenyik, J. Heidler, L. Needham and L. Goldman, Epidemiology, 2006, 17, S394-S394.

20 O. Midasch, T. Schettgen and J. Angerer, Int. J. Hyg. Environ. Health, 2006, 209, 489-496.

21 C. Y. Fei, J. K. McLaughlin, R. E. Tarone and J. Olsen, Environ. Health Perspect., 2007, 115, 1677-1682.
22 B. J. Apelberg, F. R. Witter, J. B. Herbstman, A. M. Calafat, R. U. Halden, L. L. Needham and L. R. Goldman, Environ. Health Perspect., 2007, 115, 1670-1676.

23 N. Washino, Y. Saijo, S. Sasaki, S. Kato, S. Ban, K. Konishi, R. Ito, A. Nakata, Y. Iwasaki, H. Nakazawa and R. Kishi, Environ. Health Perspect., 2009, 117, 660-667.

24 G. W. Olsen, J. L. Butenhoff and L. R. Zobel, Reprod. Toxicol., 2009, 27, 212-230

25 A. Kärrman, J. F. Mueller, B. Van Bavel, F. Harden, L. M. L. Toms and G. Lindstrom, Environ. Sci. Technol., 2006, 40, 3742-3748.

26 E. Jurado, F. M. Jaward, R. Lohmann, K. C. Jones, R. Simo and J. Dachs, Environ. Sci. Technol., 2004, 38, 5505-5513.

27 L. Ahrens, J. L. Barber, Z. Xie and R. Ebinghaus, Environ. Sci. Technol., 2009, 43, 3122-3127.

28 A. Jahnke, U. Berger, R. Ebinghaus and C. Temme, Environ. Sci. Technol., 2007, 41, 3055-3061.

29 L. M. L. Toms, A. M. Calafat, K. Kato, J. Thompson, F. Harden, P. Hobson, A. Sjodin and J. F. Mueller, Environ. Sci. Technol., 2009, 43, 4194-4199.

30 K. Kannan, S. Corsolini, J. Falandysz, G. Fillmann, K. S. Kumar, B. G. Loganathan, M. A. Mohd, J. Olivero, N. Van Wouwe, J. H. Yang and K. M. Aldous, Environ. Sci. Technol., 2004, 38, 4489-4495.

31 H. B. Röllin, T. M. Sandanger, L. Hansen, K. Channa and J. O. Odland, Sci. Total Environ., 2009, 408, 146-152.

32 H. B. Röllin, C. V. C. Rudge, Y. Thomassen, A. Mathee and J. O. Odland, J. Environ. Monit., 2009, 11, 618-627.

33 C. Rylander, T. P. Duong, J. O. Odland and T. M. Sandanger, J. Environ. Monit., 2009, 11, 2002-2008.

34 C. R. Powley, S. W. George, T. W. Ryan and R. C. Buck, Anal. Chem., 2005, 77, 6353-6358.

35 A. Karrman, I. Langlois, B. van Bavel, G. Lindstrom and M. Oehme, Environ. Int., 2007, 33, 782-788.

36 D. J. Ehresman, J. W. Froehlich, G. W. Olsen, S. C. Chang and J. L. Butenhoff, Environ. Res., 2007, 103, 176-184.

37 R. Monroy, K. Morrison, K. Teo, S. Atkinson, C. Kubwabo, B. Stewart and W. G. Foster, Environ. Res., 2008, 108, 56-62.

38 O. Midasch, H. Drexler, N. Hart, M. W. Beckmann and J. Angerer, Int. Arch. Occup. Environ. Health, 2007, 80, 643-648.

39 R. Vestergren and I. T. Cousins, Environ. Sci. Technol., 2009, 43, $5565-5575$.

40 A. M. Calafat, L. Y. Wong, Z. Kuklenyik, J. A. Reidy and L. L. Needham, Environ. Health Perspect., 2007, 115, 1596-1602.

41 J. P. Benskin, A. O. De Silva, L. J. Martin, G. Arsenault, R. McCrindle, N. Riddell, S. A. Mabury and J. W. Martin, Environ. Toxicol. Chem., 2009, 28, 542-554.

42 J. A. Bjorklund, K. Thuresson and C. A. De Wit, Environ. Sci. Technol., 2009, 43, 2276-2281.

$43 \mathrm{H}$. Fromme, S. A. Tittlemier, W. Volkel, M. Wilhelm and D. Twardella, Int. J. Hyg. Environ. Health, 2009, 212, 239-270.

44 L. S. Haug, C. Thomsen and G. Bechert, Environ. Sci. Technol., 2009, 43, 2131-2136.

45 O. S. von Ehrenstein, S. E. Fenton, K. Kato, Z. Kuklenyik, A. M. Calafat and E. P. Hines, Reprod. Toxicol., 2009, 27, 239245 .

46 A. O. De Silva and S. A. Mabury, Environ. Sci. Technol., 2006, 40, 2903-2909.

47 N. Riddell, G. Arsenault, J. P. Benskin, B. Chittim, J. W. Martin, A. McAlees and R. McCrindle, Environ. Sci. Technol., 2009, 43, $7902-7908$. 